\title{
Non-invasive assessment of cardiac output in children
}

\author{
J R Richardson, J Ferguson, J Hiscox, J Rawles
}

\begin{abstract}
Background-Stroke distance, the systolic velocity integral of aortic blood flow, is a linear analogue of stroke volume; its product with heart rate is minute distance, analogous to cardiac output.

Objective-To investigate the feasibility of assessing cardiac output in children with a simple non-invasive Doppler ultrasound technique, and to determine the normal range of values.

Methods-Peak aortic blood velocity, stroke distance, and minute distance were measured through the suprasternal window in 166 children (mean age 9.6 years, range 2-14) using a portable non-imaging Doppler ultrasound instrument.

Results-The technique was well tolerated by all the children participating. Mean peak aortic blood velocity was $138 \mathrm{~cm} / \mathrm{s}$ and was independent of age. Mean stroke distance was $31.8 \mathrm{~cm}$ and showed a small but significant increase with age; mean minute distance was $2490 \mathrm{~cm}$ and fell with age, as did heart rate.

Conclusions-Suprasternal Doppler ultrasound measurement of stroke distance is a convenient, well tolerated, non-invasive technique for the assessment of cardiac output in children. The normal range of values during childhood has been established. The technique has great potential for assessing hypovolaemia in children. $(\Im$ Accid Emerg Med 1998;15:304-307)
\end{abstract}

Keywords: stroke distance; cardiac output; Doppler; children

\section{Royal Aberdeen Children's Hospital, Aberdeen \\ J R Richardson \\ J Ferguson \\ J Hiscox}

Medicines Assessment Research Unit, University of Aberdeen J Rawles

Correspondence to: Dr Jeremy R Richardson, Accident and Emergency Department, Aberdeen Royal Hospitals NHS Trust,

Foresterhill, Aberdeen AB25 22D (e-mail:

j.r.richardson@abdn.ac.uk).

Accepted for publication 4 June 1998
Trauma causes more deaths in the first few decades of life than any disease. In 1988 a retrospective study of 1000 trauma deaths in England and Wales showed that $63 \%$ of non-head injury deaths were potentially preventable, and a major factor in many of these deaths was failure to recognise the presence or the magnitude of blood loss. ${ }^{1}$ Advanced Tauma Life Support and Advanced Paediatric Life Support courses now address this problem and provide clinicians with a system to improve the management of trauma patients. These courses emphasise the importance of heart rate, blood pressure, and other physical signs in the assessment of hypovolaemia.

It has been known for many years, however, that heart rate and blood pressure provide a poor guide to the degree of hypovolaemia in adults. In 1944 Barcroft and colleagues ven- esected 1 litre of blood from healthy volunteers while monitoring heart rate, blood pressure, central venous pressure, and cardiac output. ${ }^{2}$ Initially, heart rate increased and arterial blood pressure remained unchanged (baroreceptor reflex). After more severe blood loss ( $>15$ $20 \%$ ) both heart rate and blood pressure were shown to fall. Cardiac output and central venous pressure, however, fell immediately and progressively during the haemorrhage. The effects on the cardiovascular system of tissue damage and anxiety complicate matters further, as increased sympathetic outflow causes an increase in both heart rate and arterial blood pressure. ${ }^{3}$

For these reasons the assessment of hypovolaemia in trauma patients is difficult, but especially so in children, due to their ability to compensate after blood loss. Hypotension in the shocked child is recognised as frequently being a preterminal sign. If children were to show the same progressive fall in cardiac output after haemorrhage that is observed in adults, then a technique for assessing cardiac output would be of benefit, particularly if it is non-invasive and convenient to perform in an accident and emergency (A\&E) resuscitation room.

However, cardiac output, the volume of blood flowing out of the heart per minute, is measured only with great difficulty. The reference method is thermodilution, which is precluded in most circumstances in children because it is invasive, hazardous, and unreliable. But the possibility of assessing cardiac output simply and non-invasively is opened up by considering cardiac output as a distance rather than as a volume. ${ }^{4}$

\section{Linear cardiac output}

Cardiac output is related to the body's metabolic needs and therefore also to its size. To make meaningful comparison of stroke volume or cardiac output in different individuals it is necessary to take account of body size, which is customarily represented by body surface area. To normalise for variation of body size and metabolic requirement, stroke or minute volume is divided by body surface area, giving stroke or cardiac index, respectively:

Stroke volume/body surface area $=$ stroke index.

The use of body surface area to normalise for variation of metabolic need is not ideal, however, as a short obese individual may have the same body surface area as a tall thin person but a quite different metabolic rate and cardiac output. It may be preferable to use the area of 
Table 1 Doppler derived haemodynamic variables in 166 children

\begin{tabular}{lllll}
\hline & Peak velocity $(\mathrm{cm} / \mathrm{s})$ & Stroke distance $(\mathrm{cm})$ & Heart rate (bpm) & Minute distance $(\mathrm{cm})$ \\
\hline Mean (SD) & $137.6(21.0)$ & $31.80(5.15)$ & $78.8(14.0)$ & $2490(506)$ \\
Coefficient of variation (\%) & 15.3 & 16.2 & 17.7 & 20.3
\end{tabular}

part of the body that is representative of the whole, rather than the area of the whole body, particularly if the area chosen more closely reflects metabolic need.

There is evidence that during infancy and childhood, growth of the aorta is regulated by the velocity of blood flowing within it. ${ }^{5}$ As the metabolic need of the infant increases, so does cardiac output and the velocity of blood flow in the aorta. Peak aortic blood velocity in many animal species of widely different sizes has been shown to be just less than that which results in turbulent flow. ${ }^{6}$ Any increase in velocity, such as would occur with an increase in cardiac output to nourish a growing child, would lead to aortic blood flow becoming turbulent. It has been suggested that turbulent flow acts as the stimulus for aortic growth, restoring aortic blood velocity to its former value. As a consequence of this mechanism, the peak velocity of aortic blood flow remains remarkably constant during the first three decades of life, in spite of a several-fold increase in body size, metabolic need, and cardiac output. ${ }^{7}$ Aortic cross sectional area reflects the metabolic need of an individual, and could therefore be used instead of body surface area to normalise for variation of body size and metabolic requirement:

Stroke volume/aortic cross sectional area $=$ stroke distance.

Because aortic cross sectional area is a measure of body size that reflects metabolic need, stroke distance is analogous to stroke index. Stroke distance may be measured directly as the systolic velocity integral of aortic blood flow, and may be envisaged as the distance that a column of blood travels in the aorta during systole. The above relationship holds true because stroke volume may be calculated as the

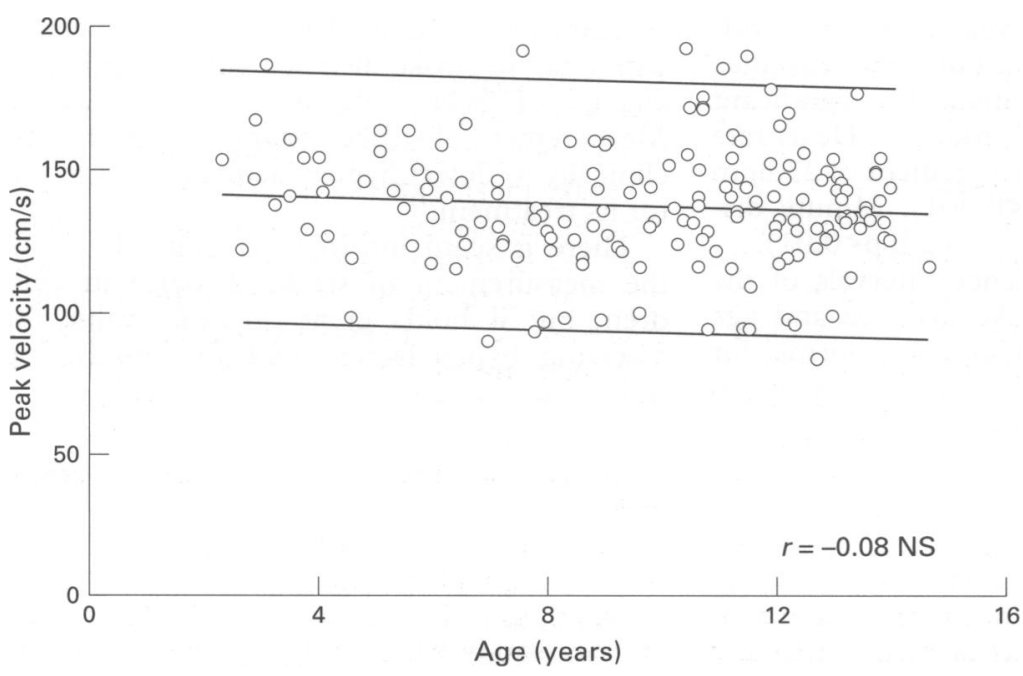

Figure 1 Peak aortic blood velocity plotted against age in years in 166 children; regression line and 95\% confidence intervals shown. product of aortic cross sectional area and stroke distance:

Stroke volume $=$ aortic cross sectional area $\times$ stroke distance.

Stroke distance may be measured simply and non-invasively using continuous wave Doppler ultrasound using the suprasternal ultrasound window onto the aortic arch; there is no need to measure aortic cross sectional area. Because stroke distance is closely related to stroke index, it would be expected to be independent of body size. A single measurement of stroke distance should provide an absolute indication of cardiac output for comparison with a previously established normal range of values; serial measurements give additional information. The product of stroke distance with heart rate is minute distance, which is analogous to cardiac index.

In adults over 30 years old, peak aortic blood velocity and stroke distance fall with age, ${ }^{8}$ but having established a normal range of values at different ages, measurement of stroke distance is an effective method of detecting haemodynamic disturbance, including hypovolaemia. The aim of this study in children is to establish the normal range for stroke distance, as a prelude to evaluating this technique in the assessment and management of hypovolaemia.

\section{Methods}

Children about to be discharged from the review clinic in the A\&E department of Royal Aberdeen Children's Hospital were invited to participate in this study, for which ethical committee approval had been obtained. The child was allowed to sit at rest for several minutes, then placed in the supine position. A portable Doppler monitor (Deltex Medical, Chichester, West Sussex) with a continuous wave $2 \mathrm{MHz}$ probe was used. The probe was placed in the suprasternal notch, directed at the arch of the aorta, and its position adjusted to give the cleanest velocity time complexes on the monitor, and the highest audible pitch. The instrument evaluates the complexes as they are recorded, and was set to take an average of 10 cardiac cycles. Measurements were made of peak velocity, stroke distance, heart rate, and minute distance.

\section{Results}

From 172 volunteers, satisfactory recordings were obtained in all but six $(97 \%)$. None of the children was distressed by the procedure.

Children of all ages from 2 to 14 were examined (mean 9.6 years). Table 1 gives mean values of haemodynamic variables in the 166 children with evaluable recordings. 


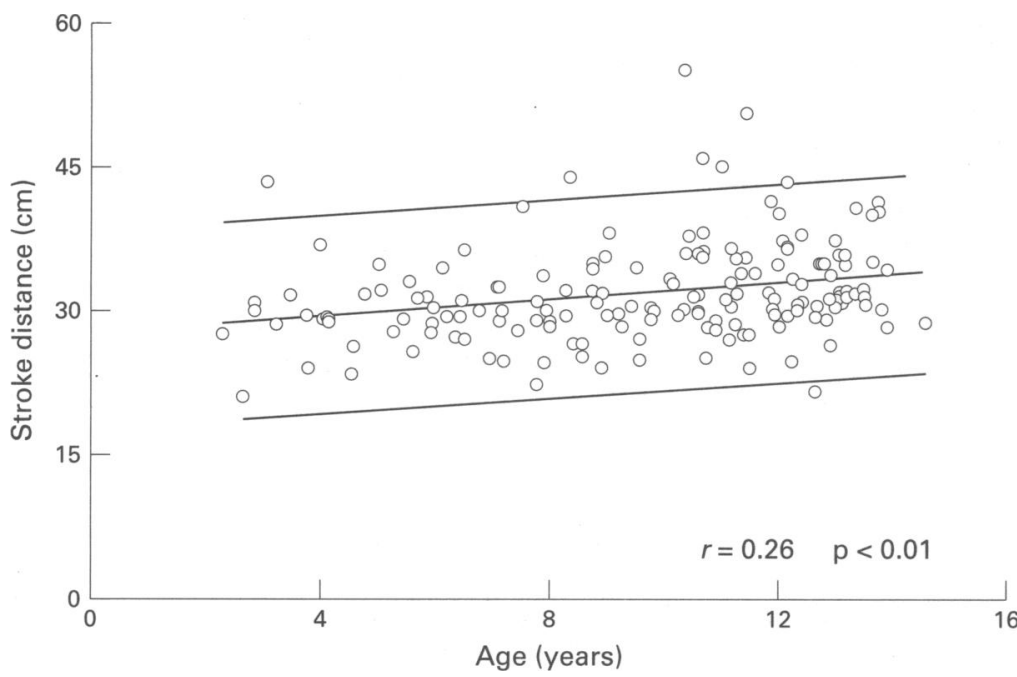

Figure 2 Stroke distance plotted against age in years in 166 children; regression line and $95 \%$ confidence intervals shown.

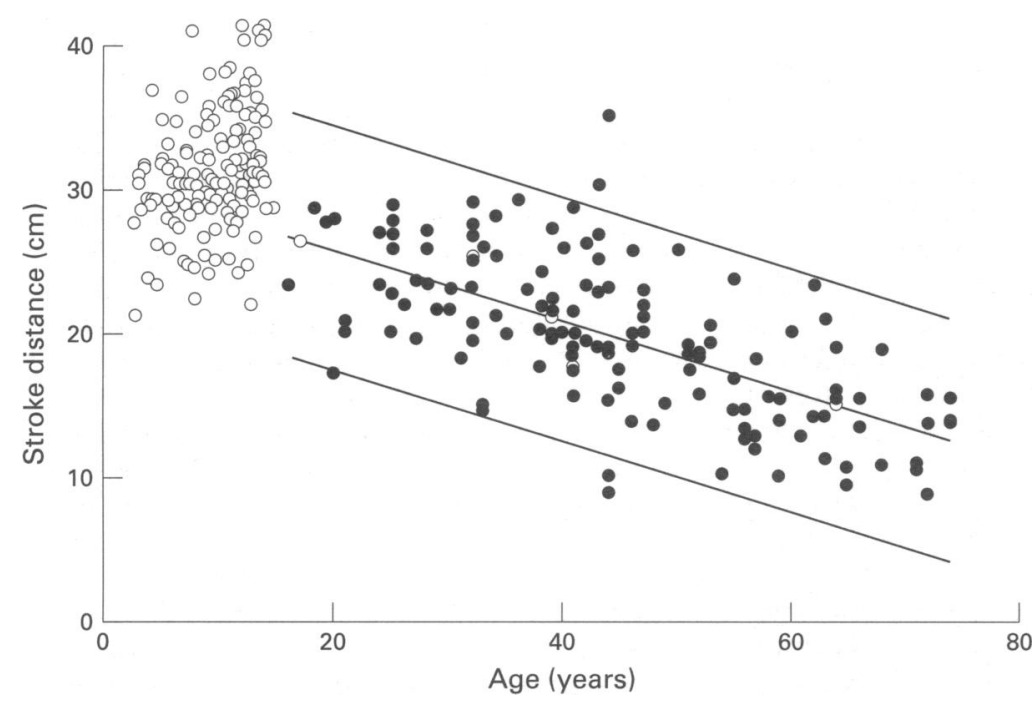

Figure 3 Stroke distance plotted against age in years in 140 adults ${ }^{8}$ (closed circles); regression line and $95 \%$ confidence intervals shown. Also shown are the stroke distance measurements from 166 children (open circles).

The effects of age on peak aortic blood velocity and stroke distance are seen in figs 1 and 2. Peak aortic blood velocity is independent of age ( $r=-0.08$; NS), but stroke distance shows a small but statistically significant increase with age $(r=0.26 ; p<0.01)$. Heart rate falls steeply with age with a gradient of $23 \mathrm{bpm}$ per decade $(r=-0.52 ; \mathrm{p}<0.001)$. Minute distance also falls with age $(r=-0.22 ; \mathrm{p}<0.01)$.

From the $95 \%$ confidence intervals of the relationship between stroke distance and age shown in fig 2, the lower limits of normal for stroke distance are 20,22 , and $24 \mathrm{~cm}$ at age 5 , 10 , and 15 years, respectively.

\section{Discussion}

In this study, mean peak aortic blood velocity in 166 children was $138 \mathrm{~cm} / \mathrm{s}$ with a coefficient of variation of $15 \%$. This is identical to the value found in a series of 120 children published by Light in 1978 (coefficient of vari- ation $13 \%$ ), although the two series were separated by 20 years and 500 miles, and the recordings were made by different equipment, and evaluated by different methods. ${ }^{7}$ Remarkably, too, in neither series is there any significant relationship between peak aortic blood velocity and age between 1 to 14 years, covering a several-fold range of body weight and height. These observations show that the measurement of peak aortic blood velocity is far from being arbitrary, and support the hypothesis that, by virtue of its effect on aortic growth, aortic blood velocity is a regulated variable.

Stroke distance is calculated as the integral of maximum instantaneous aortic blood velocity during systole. Its value depends on both peak velocity, and duration of systole, or flow time. While peak velocity is unchanged during childhood, heart rate falls and flow time increases. As a consequence, stroke distance shows a modest increase, of $13 \%$ per decade. The greatest percentage change of any of the haemodynamic variables measured is shown by heart rate, falling by $29 \%$ per decade. Minute distance, the product of stroke distance and heart rate, falls by $14 \%$ per decade, the effect of age on heart rate predominating over that on stroke distance.

In adults peak velocity and stroke distance fall with age ${ }^{8}$ due to progressive dilatation of the aorta. ${ }^{9}$ The previously published ${ }^{8}$ normal values for stroke distance in adults of different ages are shown between the $95 \%$ confidence intervals illustrated in fig 3 . The values in children are entirely consistent with those in adults, and most of the measurements of stroke distance in children fall within the projected confidence intervals for the adults.

Stroke distance has been measured in various abnormal steady states in adults, and has behaved as would be expected of a measure of cardiac output. Thus, stroke distance is increased in pregnancy and anaemia, normal in hypertension, and reduced in acute myocardial infarction and cardiac failure. ${ }^{4}$ Acute hypovolaemia simulated experimentally by lower body suction is reliably detected by measurement of stroke distance. ${ }^{3}$ In nine blood donors, venesection of one unit of blood was associated with a fall of stroke distance but no significant change of heart rate or blood pressure. ${ }^{10}$ Measurement of stroke distance has been used clinically to detect hypovolaemia and to monitor its treatment. ${ }^{11}$

There is scant information available about the measurement of stroke distance in children, but it holds great potential value for assessing hypovolaemia and for monitoring fluid resuscitation.

Conflict of interest: J Rawles has a consultancy with Deltex Medical Ltd.

1 Anderson ID, Woodford M, DeDombal FT, et al. Retrospective study of 1000 deaths from injury in England and Wales. BMJ 1988;296:1305-8.

2 Barcroft H, Edholm OG, McMichael J, et al. Post Barcroft H, Edholm OG, McMichael J, et al. Post
haemorrhagic fainting. Study by cardiac output and forearm flow. Lancet 1944;i:489-491. 
3 Little RA, Kirkman E, Driscoll P, et al. Preventable deaths after injury: why are the traditional "vital"signs poor indicators of blood loss? J Accid Emerg Med 1995;12:114.

4 Rawles JM. Linear cardiac output: the concept, its measurement, and applications. In: Chambers J, Monaghan MJ, ment, and applications. In: Chambers J, Monaghan MJ, eds. Echocardiography: an international

5 Light LH. Aortic blood velocity, the regulation of aortic growth and Doppler cardiac output. Biomedizinishe Technik 1990;33(suppl 3):115-6.

6 Parker KH. Instability in arterial blood flow. In: Hwang NHC, Norman NA, eds. Cardiovascular flow dynamics and measurements. Baltimore: University Park Press, 1977:633 63.
7 Light LH. Implications of aortic blood velocity measurements in children. J Physiol 1978;285:17-18P.

8 Mowat DHR, Haites NE, Rawles JM. Aortic blood velocity measurement in healthy adults using a simple ultrasound technique. Cardiovasc Res 1983;17:75-80.

9 Towfiq B, Weir J, Rawles JM. Effect of age and blood pressure on aortic size and stroke distance. Br Heart J 1986;55: 560-8.

10 Hanson JM, van Hoeyweghen R, Kirkman E, et al. Use of stroke distance in the early detection of simulated blood loss. J Trauma (in press)

11 Sammy I, Hanson J, James MR. Cerebral oximetry and stroke distance: the future of emergency department monitoring? J Accid Emerg Med 1996;13:313-15.

\section{EMERGENCY CASEBOOK}

\section{Closed digital artery injury}

A 55 year old right handed medically fit non-smoking farmer presented to the accident and emergency department six hours after a closed injury of the left little, ring, and middle fingers (at the level of the middle phalanx in the ring and middle finger and at the distal phalanx level of the little finger). His fingers had been by trapped by a $50 \mathrm{~kg}$ weight of metal.

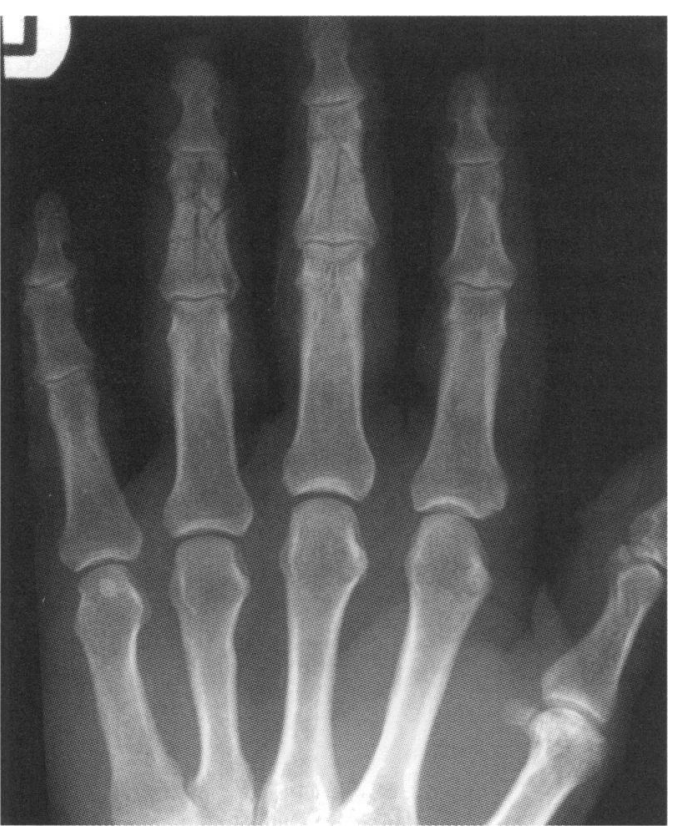
The ring finger progressively became dusky in colour and cold, while skin sensation decreased and gradually became absent at the level of the fracture. Hand $x$ ray showed a comminuted fracture of the middle phalanx of the middle finger, comminuted fracture with intra-articular extension of the middle phalanx of the ring finger, and comminuted dorsal fracture of the base of the distal phalanx of the little finger (fig 1).

Doppler study of the ring finger revealed loss of digital artery pulsation at the level of the fracture. Immediate surgical exploration of the finger through zigzag volar skin incision revealed rupture of the radial digital artery of the finger at the level of the middle phalanx. The neurovascular structures otherwise grossly looked normal. The artery was repaired by microsurgical technique.

There are two descriptions of closed arterial injury of the finger. ${ }^{12}$ Injuries of the digital arteries of the hand are usually due to open wounds. This may give a mistaken sense of security regarding arterial injury with closed fractures of the hand. Recognition of closed digital artery injury is difficult; the dilemma is that finger ischaemia may be secondary to venous congestion and will disappear with elevation, but this can result in a gangrenous finger if the digital artery is damaged. This can easily be ruled out by ultrasound. Early recognition and surgical repair can save the finger if the digital arteries are injured.

1 Lister G. The hand: diagnosis and indications, 3rd Ed. New York: Churchill Livingstone, 1993:592.

2 Giddins GEB, Burge PD. Closed digital artery injury. J Bone Joint Surg 1996:78B:325-6.

A A FARAJ, M A CRAIGEN Orthopaedic Department, University Hospital, Nottingham NG7 $2 U H$. Correspondence to: A A Faraj. 